Document downloaded from:

http://hdl.handle.net/10251/55793

This paper must be cited as:

Tirado Peláez, P. (2014). A new model based on a fuzzy quasi-metric type Baire applied to analysis of complexity. Journal of Intelligent and Fuzzy Systems. 27:2545-2550.

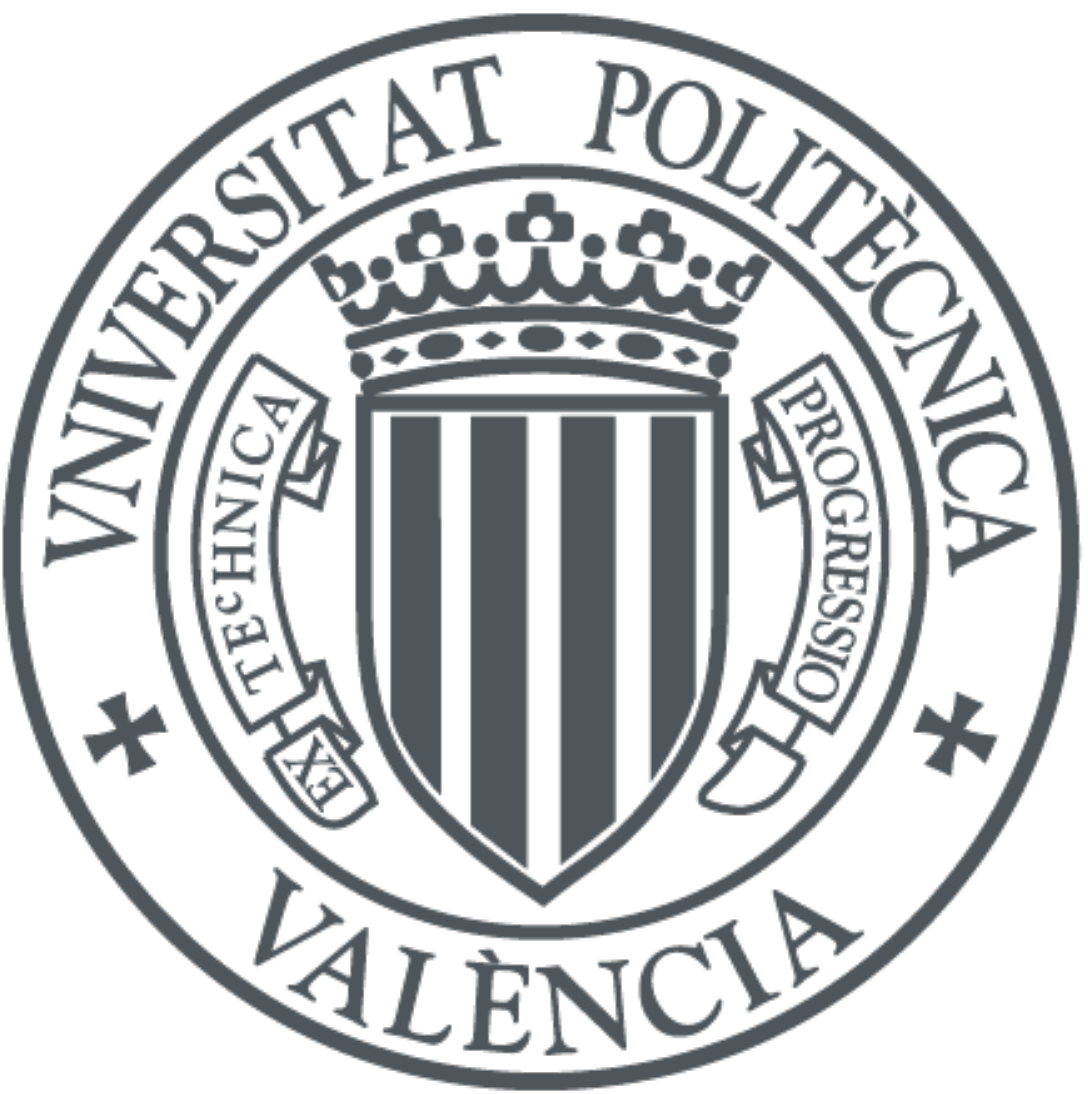

The final publication is available at

http://dx.doi.org/10.3233/IFS-141228

Copyright IOS Press

Additional Information 


\title{
A new model based on a fuzzy quasi-metric type Baire applied to analysis of complexity
}

\author{
Pedro Tirado * \\ Instituto Universitario de Matemática Pura y Aplicada \\ Universitat Politècnica de València, 46022 Valencia, Spain \\ pedtipe@mat.upv.es
}

We analyze the complexity of an expoDC algorithm by deducing the existence of solution for the recurrence inequation associated to this algorithm by means of techniques of Denotational Semantics in the context of fuzzy quasimetric spaces. The fuzzy quasi-metrics provide an additional parameter " $t$ " such that a suitable use of this ingredient gives rise to extra information on the involved computational process. This analysis is done by means of a fuzzy quasi-metric version of the Banach contraction principle on a space of partial functions endowed by a suitable adaptation of the Baire quasi-metric.

\section{Introduction}

The complexity quasi-metric space (introduced by M. Schellekens in [16]) provides an efficient tool to show, in a systematized way, the existence (and uniqueness) of solution for the recurrence equations or inequations typically associated to several distinguished kinds of algorithms for which the execution time depends on one parameter $[16,11,12,2]$, and then it is a suitable model to analyze the complexity of such algorithms. In particular, this approach was generalized in [12] to the case of expoDC algorithm for which the execution time depends on more than one parameter, which is carefully

*This research is supported by the Ministry of Economy and Competitiveness of Spain, Grant MTM2012-37894-C02-01 and by Universitat Politècnica de València, Grant PAID06-12-SP20120471. 
discussed in [1, Section 7.7], where the following recurrence inequation for this algorithm is obtained:

(1) $T(m, n) \leq\left\{\begin{array}{l}0, \quad \text { if } n=1, \\ T(m, n / 2)+L(m n / 2, m n / 2), \\ T(m, n-1)+L(m,(n-1) m), \quad \text { if } n \text { is even, }\end{array}\right.$

for all $(m, n) \in N \times N$ (where $N$ denotes the set of positive integer numbers).

According to [1, Section 7.7], $L(m, n)$ denotes the time needed to multiply two integers of sizes $m$ and $n$, and $T(m, n)$ denotes the time spent multiplying when computing $a^{n}$, where $m$ is the size of $a$, so the execution time of this algorithm depends on two parameters.

Let $L(1,1)=c>0$. Then, it is constructed in [12] a new "complexity" quasi-metric space $\left(\mathcal{C}_{0, c}, d_{0, c}\right)$, where

$$
\mathcal{C}_{0, c}=\{f: N \times N \rightarrow[0, \infty): f(m, 1)=0 \text {, and } f(m, n) \geq c \text { for } n>1\},
$$

and $d_{0, c}$ is the bicomplete quasi-metric on $\mathcal{C}_{0, c}$ given by

$$
d_{0, c}(f, g)=\sum_{m=1}^{\infty} 2^{-m}\left[\sum_{n=2}^{\infty} 2^{-n} \max \left\{\left(\frac{1}{g(m, n)}-\frac{1}{f(m, n)}\right), 0\right\}\right]
$$

The recurrence (1) induces, in a natural way, the functional $\Phi$ defined on $\mathcal{C}_{0, c}$ by

$$
\Phi f(m, n)=\left\{\begin{array}{l}
0, \quad \text { if } n=1, \\
f(m, n / 2)+L(m n / 2, m n / 2), \quad \text { if } n \text { is even, } \\
f(m, n-1)+L(m,(n-1) m), \quad \text { otherwise }
\end{array}\right.
$$

Then, it is proved in [12] that $\Phi$ is a contraction map on $\left(\mathcal{C}_{0, c}, d_{0, c}\right)$ with contraction constant $3 / 4$, and thus $\Phi$ has a unique solution $f_{0}$ which is obviously a solution for $T$.

Motivated by the usefulness of partial functions in Denotational Semantics (see [13]) we here analyze the complexity of this expoDC algorithm by deducing the existence of solutions for the recurrence inequation (1) by means of techniques of Denotational Semantics based in proving that the functional $\Phi$ above is a contraction map with contraction constant $1 / 2$ on certain space 
of partial functions endowed by an appropriate bicomplete fuzzy quasi-metric which provides an additional parameter "t" such that a suitable use of this ingredient may give rise to extra information on the involved computational process, moreover our approach provides an improvement of the contraction constant with respect to one obtained from the complexity space $\left(\mathcal{C}_{0, c}, d_{0, c}\right)$.

\section{Preliminaries}

In this section we recall some pertinent concepts and well-known facts on quasi-metric and fuzzy quasi-metric spaces.

Following the modern terminology, by a quasi-metric on a nonempty set $X$ we mean a nonnegative real valued function $d$ on $X \times X$ such that for all $x, y, z \in X:$

(i) $x=y$ if and only if $d(x, y)=d(y, x)=0$;

(ii) $d(x, z) \leq d(x, y)+d(y, z)$.

If $d$ satisfies condition (i) above and

(ii') $d(x, z) \leq \max \{d(x, y), d(y, z)\}$

then, $d$ is called a non-Archimedean quasi-metric on $X$.

If $d$ satisfies the conditions (i), (ii) and

(ii") $d(x, y)=d(y, x)$

then, $d$ is called a metric on $X$.

The notion of a non-Archimedean metric is defined in the obvious manner.

A (non-Archimedean) quasi-metric space is a pair $(X, d)$ such that $X$ is a nonempty set and $d$ is a (non-Archimedean) quasi-metric on $X$.

Each quasi-metric $d$ on $X$ generates a $T_{0}$ topology $\tau_{d}$ on $X$ which has as a base the family of open balls $\left\{B_{d}(x, r): x \in X, r>0\right\}$, where $B_{d}(x, r)=\{y \in X: d(x, y)<r\}$ for all $x \in X$ and $r>0$.

Given a (non-Archimedean) quasi-metric $d$ on $X$, then the function $d^{-1}$ defined on $X \times X$ by $d^{-1}(x, y)=d(y, x)$, is also a (non-Archimedean) quasimetric on $X$, called the conjugate of $d$, and the function $d^{s}$ defined on $X \times X$ 
by $d^{s}(x, y)=\max \left\{d(x, y), d^{-1}(x, y)\right\}$ is a (non-Archimedean) metric on $X$.

A quasi-metric space $(X, d)$ is said to be bicomplete if $\left(X, d^{s}\right)$ is a complete metric space. In this case, we say that $d$ is a bicomplete quasi-metric on $X$.

By a contraction map on a (quasi-)metric space $(X, d)$ we mean a selfmap $f$ on $X$ such that $d(f x, f y) \leq k d(x, y)$ for all $x, y \in X$, where $k$ is a constant with $0<k<1$. The number $k$ is called a contraction constant for $f$.

It is clear that if $f$ is a contraction map on a quasi-metric space $(X, d)$ with contraction constant $k$, then $f$ is a contraction map on the metric space $\left(X, d^{s}\right)$ with contraction constant $k$.

According to [17], a binary operation $*:[0,1] \times[0,1] \rightarrow[0,1]$ is a continuous t-norm if $*$ satisfies the following conditions: (i) $*$ is associative and commutative; (ii) $*$ is continuous; (iii) $a * 1=a$ for every $a \in[0,1]$; (iv) $a * b \leq c * d$ whenever $a \leq c$ and $b \leq d$, with $a, b, c, d \in[0,1]$.

Paradigmatic examples of continuous t-norm are Min, Prod, and $T_{L}$ (the Lukasiewicz t-norm).

In the following Min will be denoted by $\wedge$, Prod by $\cdot$ and $T_{L}$ by $*_{L}$. Thus we have $a \wedge b=\min \{a, b\}, a \operatorname{Prod} b=a . b$ and $a *_{L} b=\max \{a+b-1,0\}$ for all $a, b \in[0,1]$. The following relations hold:

$\wedge \geq \cdot \geq *_{L}$. In fact, $\wedge \geq *$ for any continuous t-norm $*$.

Definition 1 [5]. A KM-fuzzy quasi-metric on a (nonempty) set $X$ is a pair $(M, *)$ such that $*$ is a continuous t-norm and $M$ is a fuzzy set in $X \times X \times[0, \infty)$ such that for all $x, y, z \in X$ :

(KM1) $M(x, y, 0)=0$;

(KM2) $x=y$ if and only if $M(x, y, t)=M(y, x, t)=1$ for all $t>0$;

(KM3) $M(x, z, t+s) \geq M(x, y, t) * M(y, z, s)$ for all $t, s \geq 0$;

$(\mathrm{KM} 4) M\left(x, y,{ }_{-}\right):[0, \infty) \rightarrow[0,1]$ is left continuous.

Definition 2 [5]. A KM-fuzzy quasi-metric space is a triple $(X, M, *)$ such that $X$ is a (nonempty) set and $(M, *)$ is a KM-fuzzy quasi-metric on $X$. 
If $(M, *)$ satisfies the symmetry axiom (i.e if for all $x, y \in X$ and $t>0$, $M(x, y, t)=M(y, x, t))$, then $(M, *)$ is a fuzzy metric in the sense of Kramosil and Michalek ([7]) and $(X, M, *)$ is a fuzzy metric space in the sense of Kramosil and Michalek.

In the following, KM-fuzzy quasi-metrics and fuzzy metrics in the sense of Kramosil and Michalek will be simply called fuzzy quasi-metrics and fuzzy metrics respectively, and KM-fuzzy quasi-metric spaces and fuzzy metric spaces in the sense of Kramosil and Michalek will be simply called fuzzy quasi-metric spaces and fuzzy metric spaces, respectively.

If $(M, *)$ is a fuzzy quasi-metric on $X$, then $\left(M^{-1}, *\right)$ is also a fuzzy quasi-metric on $X$, where $M^{-1}$ is the fuzzy set in $X \times X \times[0, \infty)$ defined by $M^{-1}(x, y, t)=M(y, x, t)$. Moreover, if we denote by $M^{i}$ the fuzzy set in $X \times X \times[0, \infty)$ given by $M^{i}(x, y, t)=\min \left\{M(x, y, t), M^{-1}(x, y, t)\right\}$, then $\left(M^{i}, *\right)$ is a fuzzy metric on $X[5]$.

Given a fuzzy quasi-metric space $(X, M, *)$ we define the open ball $B_{M}(x, r, t)$, for $x \in X, 0<r<1$, and $t>0$, as the set $B_{M}(x, r, t)=\{y \in X$ : $M(x, y, t)>1-r\}$. Obviously, $x \in B_{M}(x, r, t)$.

For each $x \in X, 0<r_{1} \leq r_{2}<1$ and $0<t_{1} \leq t_{2}$, we have $B_{M}\left(x, r_{1}, t_{1}\right) \subseteq$ $B_{M}\left(x, r_{2}, t_{2}\right)$. Consequently, we may define a topology $\tau_{M}$ on $X$ as

$$
\tau_{M}:=\left\{A \subseteq X: x \in \text { Athere are } r \in(0,1), t>0, \text { with } B_{M}(x, r, t) \subseteq A\right\}
$$

Moreover, for each $x \in X$ the collection of open balls $\left\{B_{M}(x, 1 / n, 1 / n)\right.$ : $n=2,3 \ldots\}$, is a local base at $x$ with respect to $\tau_{M}$. It is clear, that for any fuzzy quasi-metric space $(X, M, *), \tau_{M}$ is a $T_{0}$ topology.

The topology $\tau_{M}$ is called the topology generated by the fuzzy quasimetric space $(X, M, *)$. It is clear that each open ball $B_{M}(x, r, t)$ is an open set for the topology $\tau_{M}$.

A sequence $\left\{x_{n}\right\}_{n}$ in a fuzzy (quasi-)metric space $(X, M, *)$ converges to a point $x \in X$ with respect to $\tau_{M}$ if and only if $\lim _{n} M\left(x, x_{n}, t\right)=1$, for all $t>0$. 
By using the notion of a fuzzy metric space in the sense of Kramosil and Michalek [7], Grabiec proved in [4] a fuzzy version of the celebrated Banach fixed point theorem. To this end, Grabiec introduced the following notions: A sequence $\left\{x_{n}\right\}_{n}$ in a fuzzy metric space $(X, M, *)$ is called G-Cauchy if for each $\varepsilon \in(0,1), p \in N, t>0$ there exists $n_{0} \in N$ such that $M\left(x_{n}, x_{n+p}, t\right)>1-\varepsilon$ for all $n \geq n_{0}$. A fuzzy metric space $(X, M, *)$ is called G-complete provided that every G-Cauchy sequence in $X$ is convergent. In this case, $(M, *)$ is called a $\mathrm{G}$-complete fuzzy metric on $X$.

George and Veeramani presented in [3] an example which shows that Grabiec's notion of completeness is very strong; indeed, the fuzzy metric induced by the Euclidean metric is not complete in the sense of Grabiec. Due to this fact, they modified the definitions of Cauchy sequence and completeness due to Grabiec as follows: A sequence $\left\{x_{n}\right\}_{n}$ in a fuzzy metric space $(X, M, *)$ is called a Cauchy sequence if for each $\varepsilon \in(0,1), t>0$ there exists $n_{0} \in N$ such that $M\left(x_{n}, x_{m}, t\right)>1-\varepsilon$ for all $n, m \geq n_{0}$. A fuzzy metric space $(X, M, *)$ is called complete provided that every Cauchy sequence in $X$ is convergent. In this case, $(M, *)$ is called a complete fuzzy metric on $X$. Nevertheless the notion of G-completeness is very interesting in the case of non-Archimedean fuzzy metric spaces because (see [10, Theorem 3]) each complete non-Archimedean fuzzy metric space is G-complete.

In [10], Romaguera, Sapena and Tirado generalized Grabiec's theorem to the fuzzy quasi-metric setting. To this end they gave the following notions: A sequence $\left\{x_{n}\right\}_{n}$ in a fuzzy quasi-metric space $(X, M, *)$ is called G-Cauchy if $\left\{x_{n}\right\}_{n}$ is a G-Cauchy sequence in $\left(X, M^{i}, *\right)$. A fuzzy quasi-metric space $(X, M, *)$ is called G-bicomplete if $\left(X, M^{i}, *\right)$ is a G-complete fuzzy metric space. In this case, $(M, *)$ is called a G-bicomplete fuzzy quasi-metric on $X$. So the notions of Cauchy sequence and bicomplete fuzzy quasi-metric space can be given in a natural way as follows: A sequence $\left\{x_{n}\right\}_{n}$ in a fuzzy quasi-metric space $(X, M, *)$ is called Cauchy if $\left\{x_{n}\right\}_{n}$ is a Cauchy sequence in $\left(X, M^{i}, *\right)$. A fuzzy quasi-metric space $(X, M, *)$ is called bicomplete if $\left(X, M^{i}, *\right)$ is a complete fuzzy metric space. In this case, $(M, *)$ is called a bicomplete fuzzy quasi-metric on $X$.

Therefore, the classical Banach contraction principle can be generalized to the fuzzy quasi-metric setting as follows (see for instance [20]). 
Theorem 1 [20]. Let $(X, M, *)$ be a G-bicomplete fuzzy quasi-metric space. If $f$ is a self-map on $X$ such that there is $k \in(0,1)$ satisfying

$$
M(f x, f y, t) \geq 1-k+k M(x, y, t)
$$

for all $x, y \in X$ and $t>0$, then $f$ has a unique fixed point.

The notion of a non-Archimedean fuzzy metric space was introduced by Sapena [15]. A natural generalization of this concept to the quasi-metric setting can be found in [10] as follows: A fuzzy quasi-metric space $(X, M, *)$ such that $M(x, y, t) \geq \min \{M(x, z, t), M(z, y, t)\}$ for all $x, y, z, \in X, t>0$, is called a non-Archimedean fuzzy quasi-metric space, and $(M, *)$ is called a non-Archimedean fuzzy quasi-metric.

\section{A fuzzy quasi-metric on a space of partial functions}

In this section we construct our general framework based on a space of partial functions endowed by a fuzzy quasi-metric type Baire and analyze its completeness. We also obtain a version of Theorem 1 that we shall apply in the next section to deduce the complexity of expoDC algorithm.

In the sequel, given a nonempty alphabet $\Sigma$, we shall denote by $\Sigma^{\infty}$ the domain of (finite and infinite) words over $\Sigma$, and by $\ell(x)$ we denote the length of the word $x$. The common prefix of $x$ and $y$ is denoted by $x \sqcap y$, and if $x$ is a prefix of $y$ we write $x \sqsubseteq y$.

The so-called Baire quasi-metric (see, for instance, [14]) is the quasimetric $d_{\sqsubseteq}$ on $\Sigma^{\infty}$ given by $d_{\sqsubseteq}(x, y)=0$ if $x \sqsubseteq y$, and $d_{\sqsubseteq}(x, y)=2^{-\ell(x \sqcap y)}$ otherwise.

Note that $\left(d_{\sqsubseteq}\right)^{s}$ is the Baire metric on $\Sigma^{\infty}$.

Following [13], put $N_{\neg}=\{\{1, \ldots, n\}: n \in N\} \cup N$, and $\mathcal{P}=\{f$ : $\left.N \times B \rightarrow[0, \infty), B \in N_{\rightarrow}\right\}$.

For each $f \in \mathcal{P}$ and each $m \in N$, we define $f(m): B \rightarrow[0, \infty)$ as $f(m)(n)=f(m, n)$ for all $n \in B$ and $\mathcal{P}_{m}=\{f(m), f \in \mathcal{P}\}$. 
If $B$ is finite then $f(m)$ is a partial function.

Note also that $f(m)$ can be considered as an element of $\Sigma^{\infty}$ when $\Sigma=$ $[0, \infty)$.

Moreover $\ell(f(l))=\ell(f(m))$ for all $l, m \in N$, and $\ell(f(m))=\infty$ if and only if $B=N$.

Now we construct, for each $m \in N$ the function $M_{m}$ on $\mathcal{P}_{m} \times \mathcal{P}_{m}$ given in the following way:

$$
\begin{aligned}
& M_{m}\left(f_{m}, g_{m}\right)=1 \quad \text { if } f_{m} \text { is a prefix of } g_{m}, \\
& M_{m}\left(f_{m}, g_{m}\right)=1-d_{\sqsubseteq}\left(f_{m}(n), g_{m}(n)\right)=1-2^{-\ell\left(f_{m} \sqcap g_{m}\right)} \quad \text { if } f_{m} \text { is not a }
\end{aligned}
$$
prefix of $g_{m}$.

We can extend $M_{m}$ on $\mathcal{P} \times \mathcal{P}$ in the following way:

$$
M_{m}(f, g)=M_{m}\left(f_{m}, g_{m}\right)
$$

Now, we define $M: \mathcal{P} \times \mathcal{P} \times[0, \infty) \rightarrow[0,1]$ as it follows:

$$
\begin{aligned}
M(f, g, 0) & =0, \\
M(f, g, t) & =\inf _{m \geq t} M_{m}(f, g), m \in N
\end{aligned}
$$

for all $f, g \in \mathcal{P}$ and $t>0$. Then, we have the following theorem:

Theorem 2. ( $\mathcal{P}, M, \wedge)$ is a non-Archimedean fuzzy quasi-metric space.

Proof. It is obvious that $M$ is a fuzzy set on $\mathcal{P} \times \mathcal{P} \times[0, \infty)$ which satisfies the following conditions for all $f, g \in \mathcal{P}$ :

$M(f, g, 0)=0$,

$M(f, g, t)=M(g, f, t)=1$ for all $t>0$ if and only if $f=g$,

$M\left(f, g,_{-}\right)$is left continuous.

Let us see that for all $f, g, h \in \mathcal{P}, t>0$ :

$$
M(f, g, t) \geq \min \{M(f, h, t), M(h, g, t)\} .
$$

Indeed, we have: 


$$
\begin{aligned}
M(f, g, t) & =\inf _{m \geq t} M_{m}(f, g,) \geq \inf _{m \geq t}\left(M_{m}(f, h) \wedge M_{m}(h, g)\right) \\
& =\inf _{m \geq t} M_{m}(f, h) \wedge \inf _{m \geq t} M_{m}(h, g)=\min \{M(f, h, t), M(h, g, t)\}
\end{aligned}
$$

so $(\mathcal{P}, M, \wedge)$ is a non-Archimedean fuzzy quasi-metric space.

In [13] the following bicomplete quasi-metric is defined on $\mathcal{P}$ :

$$
d_{\mathcal{P}}(f, g)=\sup _{m \in N} d_{\sqsubseteq}(f(m), g(m)),
$$

It is easy to see that $M(f, g, t)=1-d_{\mathcal{P}}(f, g)$ it $t \in(0,1]$, so (see [21, Remark 3.9] and [10, Proposition 1]) the topologies $\tau_{M}$ and $\tau_{d_{\mathcal{P}}}$ are the same, hence $(\mathcal{P}, M, \wedge)$ is a bicomplete fuzzy quasi-metric space. Because each bicomplete non-Archimedean fuzzy quasi-metric space is G-bicomplete ([10, Lemma 1]) we deduce that $(\mathcal{P}, M, \wedge)$ is G-bicomplete.

From Theorem 1 and [21, Remark 3.9] we have:

Theorem 3. Let $(X, M, *)$ be a G-bicomplete fuzzy quasi-metric space. If $f$ is a self-mapping on $X$ such that there is $k \in(0,1)$ satisfying

$$
M(f x, f y, t) \geq 1-k+k M(x, y, t)
$$

for all $x, y \in X$ and $t \in(0, \varepsilon)$, with $\varepsilon>0$, then $f$ has a unique fixed point.

\section{Application to the complexity analysis of expoDC algorithm}

We shall prove that the recurrence inequation associated to this expoDC algorithm gives rise to a contraction map on $(\mathcal{P}, M, \wedge)$ in the sense of Theorem 3 , so the contraction map has a unique fixed point, and then the complexity of the algorithm is represented via this element.

Example. Let $\Phi: \mathcal{P} \rightarrow \mathcal{P}$ be the extension to $\mathcal{P}$ of the functional associated to the recurrence inequation of the complexity analysis of expoDC 
algorithm. Next we show that $\Phi$ is a contraction (in the sense of Theorem 3) on the G-bicomplete non-Archimedean fuzzy quasi-metric space $(\mathcal{P}, M, \wedge)$, with contraction constant $1 / 2$.

To this end, we first note that, by construction, given $m \in N$, we have $\ell\left(\Phi\left(f_{m}\right)\right) \geq \ell\left(f_{m}\right)+1$ for all $f_{m} \in \mathcal{P}_{m}$ (in particular, $\ell\left(\Phi\left(f_{m}\right)\right)=\infty$ whenever $\left.\ell\left(f_{m}\right)=\infty\right)$.

Furthermore, it is clear that

$$
f_{m} \sqsubseteq g_{m} \Longleftrightarrow \Phi\left(f_{m}\right) \sqsubseteq \Phi\left(g_{m}\right),
$$

and consequently

$$
\Phi\left(f_{m} \sqcap g_{m}\right) \sqsubseteq \Phi\left(f_{m}\right) \sqcap \Phi\left(g_{m}\right)
$$

for all $f_{m}, g_{m} \in \mathcal{P}_{m}$. Hence

$$
\ell\left(\Phi\left(f_{m}\right) \sqcap \Phi\left(g_{m}\right)\right) \geq \ell\left(\Phi\left(f_{m} \sqcap g_{m}\right)\right) \geq \ell\left(f_{m} \sqcap g_{m}\right)+1
$$

for all $f_{m}, g_{m} \in \mathcal{P}_{m}$.

For each $m \in N$ we have:

$$
\begin{aligned}
M_{m}(\Phi f, \Phi g) & =M_{m}\left(\Phi f_{m}, \Phi g_{m}\right)=1-2^{-\ell\left(\Phi f_{m} \sqcap \Phi g_{m}\right)} \\
& \geq 1-2^{-\ell\left(\Phi\left(f_{m} \sqcap g_{m}\right)\right)} \geq 1-2^{-\left(\ell\left(f_{m} \sqcap g_{m}\right)+1\right)} \\
& =1-\frac{1}{2} 2^{-\ell\left(f_{m} \sqcap g_{m}\right)} \\
& =1-\frac{1}{2}+\frac{1}{2} M_{m}(f, g)
\end{aligned}
$$

for all $f, g \in \mathcal{P}$.

So, we have:

$$
\begin{aligned}
M(\Phi f, \Phi g, t) & =\inf _{m \geq 1} M_{m}(\Phi f, \Phi g) \geq \inf _{m \geq 1}\left(1-\frac{1}{2}+\frac{1}{2} M_{m}(f, g)\right) \\
& =1-\frac{1}{2}+\frac{1}{2} M(f, g, t)
\end{aligned}
$$

for all $t \in(0, \varepsilon)$, with $0<\varepsilon<1$, and for all $f, g \in \mathcal{P}$. Hence $\Phi$ is a contraction on the G-bicomplete non-Archimedean fuzzy quasi-metric space 
$(\mathcal{P}, M, \wedge)$, with contraction constant $1 / 2$, and the conditions of Theorem 3 are satisfied, therefore $\Phi$ has a unique fixed point $f_{0}$.

Now we claim that $f_{0}$ represents the complexity of this algorithm. Indeed, by construction if $g$ satisfies this inequation then $\Phi g$ satisfies this inequation too, so we have

$$
g \leq \Phi g \leq \Phi \Phi g \leq \ldots \leq \Phi^{n} g
$$

so

$$
g \leq \lim _{n \rightarrow \infty} \Phi^{n} g=f_{0}
$$

i.e

$$
g \leq f_{0}
$$

Finally we shall deduce the known fact that $f_{0} \in \mathcal{O}\left(m^{2} n^{2}\right)$ [1, Section 7.7].

Indeed, since $L(m, n) \in \mathcal{O}(m n)$ and $L(1,1)=c,([1])$ it follows that there exist $K \geq c$ and $n_{0}>1$ such that $L(m, n) \leq K m n$ for all $m, n \geq n_{0}$. Now define a function $h \in \mathcal{P}$ by $h(m, 1)=0$, and $h(m, n)=K n_{0}^{2} m^{2} n^{2}$ whenever $n>1$. An easy computation, taking into account that $L$ is monotone increasing, shows that $\Phi h \leq h$. Since $\Phi$ is also monotone increasing we obtain that $\Phi^{n} h \leq h$, for all $n \in N$, so $f_{0}=\lim _{n \rightarrow \infty} \Phi^{n} h \leq h$ and $f_{0} \leq h$. We conclude that $f_{0} \in \mathcal{O}\left(m^{2} n^{2}\right)$.

\section{Conclusions}

We introduce a new way to analyze the complexity of expoDC algorithm by means of fixed point techniques on a space of partial functions endowed by a fuzzy quasi-metric type Baire. The fact of working with partial functions yields a more visual application of these techniques, moreover this framework provides a suitable model to indicate if the "information" contained in an element $f$ is also contained in other $g$ from a determinate input. Indeed, if $f, g \in \mathcal{P}$ and $f_{m} \sqsubseteq g_{m}$ for all $m \geq m_{0}$ then there exists $t_{0} \in\left(m_{0}-1, m_{0}\right]$ such that $M(f, g, t)=1$ for all $t>t_{0}$. Reciprocally if we compute the fuzzy quasimetric in $(f, g, t), t \in\left(m_{0}-1, m_{0}\right]$ and obtain that $M(f, g, t)=1$, it follows 
that $f_{m} \sqsubseteq g_{m}$ for all $m \geq m_{0}$, so from the input $m_{0}$ the "information" contained in $f$ is also contained in $g$. Note that others models based on quasi-metrics are not able to detect this situation. (See for instance [12]). In our future research, we will intend to apply this approach to analyze the complexity of algorithms whose execution time depends on more than two parameters or algorithms defined as a finite systems of procedures and to establish new fixed point theorems for contraction maps induced by this class of algorithms in the context of fuzzy quasi-metric spaces.

\section{References}

[1] G. Brassard, P. Bratley, Fundamentals of Algorithms, Prentice Hall, 1996.

[2] L.M. García-Raffi, S. Romaguera and M. Schellekens, Applications of the complexity space to the General Porbabilistic Divide and Conquer Algorithms, J. Math. Anal. Appl. 348 (2008) 346-355.

[3] A. George, P. Veeramani, On some results in fuzzy metric spaces, Fuzzy Sets and Systems 64 (1994), 395-399.

[4] M. Grabiec, Fixed points in fuzzy metric spaces, Fuzzy Sets and Systems 27 (1988), 385-389.

[5] V. Gregori, S. Romaguera, Fuzzy quasi-metric spaces, Appl. Gen. Topology 5 (2004), 129-136.

[6] V. Gregori, A. Sapena, On fixed point theorems in fuzzy metric spaces, Fuzzy Sets and Systems 125 (2002), 245-253.

[7] I. Kramosil, J. Michalek, Fuzzy metrics and statistical metric spaces, Kybernetika 11 (1975), 336-344.

[8] S.G. Matthews, Partial metric topology, in: Procedings 8th Summer Conference on General Topology and Applications, Ann. New York Acad. Sci. 728 (1994) 183-197.

[9] D. Mihet, A Banach contraction theorem in fuzzy metric spaces, Fuzzy Sets and Systems 144 (2004), 431-439. 
[10] S. Romaguera, A. Sapena, P. Tirado, The Banach fixed point theorem in fuzzy quasi-metric spaces with application to the domain of words, Topology Appl 154 (2007), 2196-2203.

[11] S. Romaguera and M. Schellekens, Quasi-metric properties of complexity spaces, Topology Appl. 98 (1999) 311-322.

[12] S. Romaguera, M. Schellekens, P. Tirado and O. Valero, Contraction maps on complexity spaces and expoDC algorithms, in: Proc. International Conference of Computational Methods in Sciences and Engineering ICCMSE 2007. AIP Conference Proceedings. 963 (2007) 1343-1346.

[13] S. Romaguera, P. Tirado and O. Valero, Contraction maps on spaces of partial functions endowed by the Baire quasi-metric and expoDC algorithms, in:Proc,. International Conference on Computational and Mathematical Methods in Science and Engineering, CMMSE 2010.

[14] J. Rodríguez-López, S. Romaguera, O. Valero, Denotational semantics for programming languages, balanced quasi-metrics and fixed points, International Journal of Computer Mathematics 85 (2008) 623-630.

[15] A. Sapena, A contribution to the study of fuzzy metric spaces, Appl. Gen. Topology 2 (2001), 63-76.

[16] M. Schellekens, The Smyth completion: a common foundation for denonational semantics and complexity analysis, Electronic Notes Theoret. Comput. Sci. 1 (1995) 535-556.

[17] B. Schweizer, A. Sklar, Statistical metric spaces, Pacific J. Math. 10 (1960), 314-334.

[18] B. Schweizer, A. Sklar, Probabilistic metric spaces, North-Holland, Amsterdam, 1983.

[19] P. Tirado, Contractive Maps on Complexity Analysis in Fuzzy Quasimetric Spaces, Ph.D. Thesis, Universidad Politécnica de Valencia, 2008.

[20] P. Tirado, Some remarks on the fixed point theory for fuzzy (quasi)metric spaces, Proceedings of the Workshop in Applied Topology WiAT'10, pp. 159-167. 
[21] P. Tirado, On compactness and G-completeness in fuzzy metric spaces, Iranian Journal of Fuzzy Systems, 9(4) 2012, 151-158. 